\title{
Influence Estimation of the Voltage Value on the Measurement Results for the Optical Radiation Generated by Partial Discharges on Bushing Isolator
}

\author{
P. FRĄCZ*
}

Faculty of Electrical Engineering, Automatic Control and Informatics, Opole University of Technology

S. Mikołajczyka 5, 45-271 Opole, Poland

The subject area of the paper concerns determination of possibility and application scope suggestion for the optical spectrophotometry method in the linear isolators diagnosis. Measurements results of the optical radiation emitted by partial discharges generated on a model of bushing isolator, which was placed in the air, are presented in the paper. Recording was done by use of a spectrophotometer that enabled for measurement of optical radiation in the range from 250 to $1700 \mathrm{~nm}$. The main aim of laboratory research works performed was to estimate the influence of the supply voltage value changes in range from 0 to $60 \mathrm{kV}$ on the intensity and character of the optical radiation spectrum emitted by surface partial discharges occurring on the ceramic insulating surface of the bushing isolator. In the scope of research works, analysis of voltage value changes influence on the gathered spectra was performed while its accretion and decrease was investigated. Furthermore, the measurements and analysis performed enclose the influence estimation of the time, when the voltage was attached to the high voltage electrode, on the proceeding of the optical phenomena connected with generation of surface partial discharges. In addition a comparative analysis of measurements results gathered for two designed and implemented models of bushing insulators, which differ in geometric dimensions, was performed.

PACS: 43.58.-e, 43.60.-c, 43.60.Bf

\section{Introduction}

In recent years, the non-destructive testing including the optical spectral diagnostics, which is based on measurements and analysis of generated by electric discharges electromagnetic radiation in the wavelength from $10 \mathrm{~nm}$ to $30 \mathrm{~mm}$, is gaining more and more significance in the diagnosis of high power insulation systems of electric power devices. Based on literature reports [1-11] it can be concluded that at an adequate regime of metrology, the optical spectrophotometry method not only enables for accurate detection of radiation generated by electrical discharges, but also for widely understood analysis.

Necessity to maintain high precision during diagnostic tests, as well as the computational complexity of the procedures related to results analysis, are often the reasons for little interest in application of this method for assessment of the degree of risk for electrical discharges occurrence in insulation systems. However, measurements results reported in the literature confirm the high sensitivity of this method, revealing by changes of model parameters and tests conditions. Thus it becomes possible to study a number of phenomena inaccessible by application of conventional diagnostics for insulation systems of electric power devices such as the electric, gas chromatography and the acoustic emission methods [1-11].

In operation practice there exists a big number of insulation systems constructed in such a way that solid dielectrics cooperate with liquid and gas dielectrics. The

* e-mail: p.Frącz@gmail.com plane dividing the cooperating dielectrics is usually the place in which electric strength of the whole system is the smallest. Surface electrical partial or disruptive discharges, leading to insulation breakdown, can occur along this surface. The formation and development of surface partial discharges (SPDs) depends mainly on the type and condition of the solid dielectric surface, type of the voltage supplying a system and spatial distribution of electric field intensity. The resistance of a solid dielectric to partial discharges (PDs) formation on its surface is also significant. These properties are conditioned by the internal structure of a solid dielectric, and, first of all, by the presence of a dirt layer on its surface. Dielectrics such as glass or porcelain, contrary to dielectrics of the organic origin, do not participate actively in PDs formation and development [1-11].

A bushing insulator is a classic example of a diagonally layered system. Voltage distribution in such an insulation system depends, first of all, on the surface capacity and dielectric capacity placed between the electrodes.

The bushing isolator (bushing) is a structural system allowing for carriage through walls, or partitions, or in distribution stations through tanks and through grounded transformer covers, capacitors, generators, shunt transformers or switches, of one or a few wires and for isolating them from this partition, wherein the mounting (a collar or a clamper) to the barrier is part of the bushing [5, 12-17].

In general the bushings can be divided into substation and apparatus isolators. Depending on the application, operating conditions and function, one can dis- 
tinguish between following realizations: indoor, outdoor and overhead-oil [5, 12-17].

The aim of research works, results of which are presented in this paper, was to measure and analyze the optical radiation generated by surface PDs occurring on a bushing isolator model. The scope of measurements performed included influence estimation of supply voltage changes from 0 to $0.98 U_{\mathrm{b}}$ (the breakdown voltage) on the intensity and character of optical radiation spectra emitted by SPDs. In the scope of works carried out under laboratory conditions the effect of voltage changes on spectra obtained was studied, wherein voltage reduction and increase were investigated. Moreover, measurements and analysis performed included evaluation of the impact of voltage application to the high-voltage electrode duration time (from 0 to $25 \mathrm{~min}$ ) on the optical phenomena behavior.

\section{Characteristics of the measuring system, the isolator model and the measuring conditions}

For measuring of optical radiation emitted by SPDs generated in a system modeling the bushing isolator, a spectrophotometer was applied, whose optical transducer was placed in a specially made holder attached to a tripod. The tripod used enabled for adjustment of the height, angle and distance between the measuring head and the test isolator. An AVS-USB2000 spectrometer by the firm AVANTES (Fig. 1) was used for measurements, the main element of which is a multistage diffraction grating which enables the analysis of the spectrum in the range $270-1600 \mathrm{~nm}$ with a resolution of about $0.5 \mathrm{~nm}$. A signal is delivered to the grating by a light pipe. After splitting, the radiation falls on the CCD matrix (SonyILX511). The integration time can be changed in the range from $3 \mathrm{~ms}$ to $60 \mathrm{~s}[5,12,14]$.

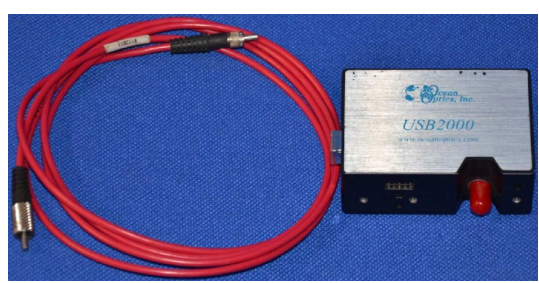

Fig. 1. AVS-USB2000 spectrophotometer by the firm AVANTES.

The measurement in the particular element of the CCD matrix (2048 elements sized $\left.12.5 \times 200 \mu \mathrm{m}^{2}\right)$ is based on the photon counts in a time unit. One count corresponds with the activation by 86 photons, which is the equivalent of the sensitivity of $2.9 \times 10^{-17} \mathrm{~J} /$ pulse. The relative sensitivity depends on the length of the wave analyzed. The root-mean-square value at the dark current is from 2.5 to 4 counts. Detailed characteristics of the spectrophotometer applied, is presented, among others, in $[5,12,13,15-16]$. The standard uncertainty of the intensity (counts quantity) specified within the type A method ranged from 10 to 100 depending on the wavelength, system configuration, voltage value and measurement time. For SPD generation an isolator model in form of porcelain sleeve filled with transformer oil was used (Fig. 2).

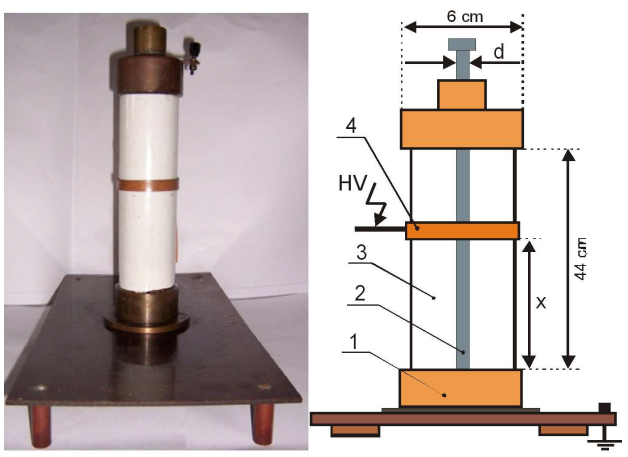

Fig. 2. View and idea diagram of the system modeling bushing isolator, where: 1 - grounded ferrule, $2-$ metal insert, 3 - porcelain insulation, 4 - movable grip.

The isolator model tested was supplied with an assay system that allowed for smooth regulation of voltage in the range from 0 to $60 \mathrm{kV}$. The assay system consisted of a control panel containing an autotransformer (5), over current protection (4) and digital voltmeter for measuring the voltage on the low voltage side (6). The voltage regulated by the autotransformer was provided to a single phase transformer type TP60, of gear 230/60000 V/ $\mathrm{V}(7)$, which has powered, through a water resistor (8), the bushing isolator modeling systems (10). The magnitude of the supply voltage on the high-voltage side was measured with an electrostatic voltmeter (9), which was placed after the resistor limiting short-circuits current (8). Schematic of the high-voltage assay system applied is presented in Fig. $3[5,14,15]$.

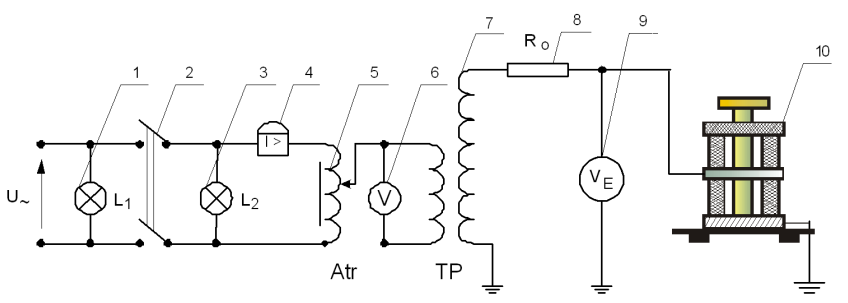

Fig. 3. Schematic of the power setup, where: 1, 3 lamps control, 2 - switch, 4 - over-current relay, 5 autotransformer, 6 - digital voltmeter, 7 - test transformer, 8 - protective resistor, 9 - electrostatic voltmeter, 10 - system modeling bushing isolator.

During each measurements series a constant distance of $3 \mathrm{~cm}$ between the measuring head of the spectrophotometer and the location of SPDs generation was maintained. Measurements have been performed in a darkened room without access to any additional sources of 
light radiation. For the results obtained being subjected to comparative analysis, the optical radiation generated by SPDs was measured at the same distance between electrodes (the $2 \mathrm{nd}$ and $3 \mathrm{rd}$ one) equal to $9 \mathrm{~cm}$ and the same distance between the spectrophotometer and SPDs generation location.

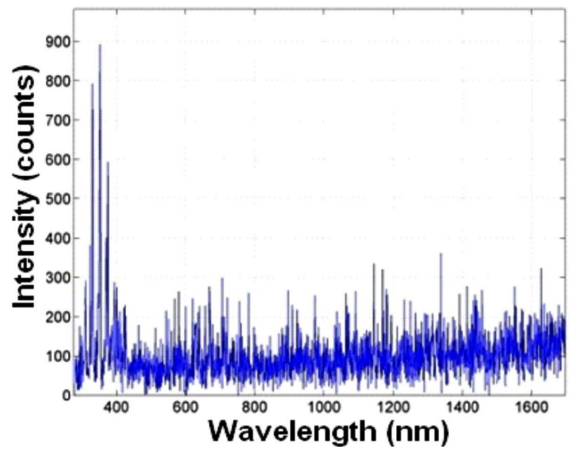

Fig. 4. Spectrum of optical radiation generated by SPDs on the model of bushing isolator at increase voltage $U=0.98 U_{\mathrm{b}}$ for the range $(270-1700) \mathrm{nm}$.

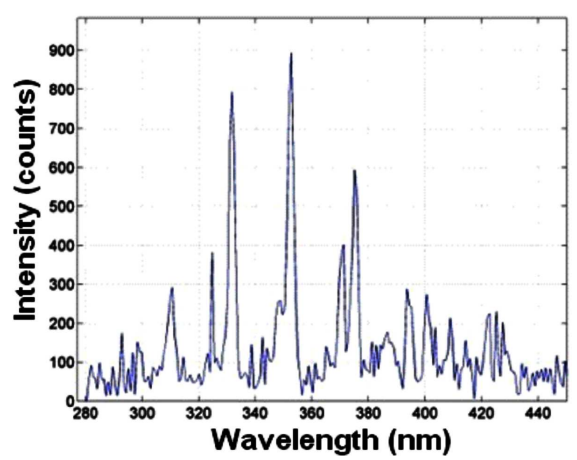

Fig. 5. Spectrum of optical radiation generated by SPDs on the model of bushing isolator at increase voltage $U=0.98 U_{\mathrm{b}}$ for the range $(270-450) \mathrm{nm}$.

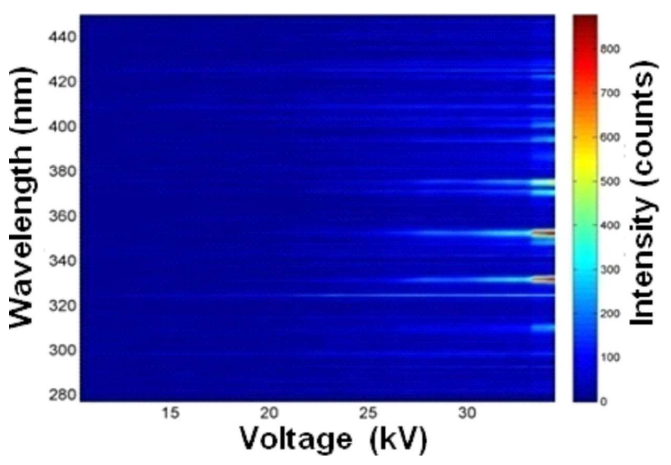

Fig. 6. Changing the intensity of optical radiation generated by the SPDs on the model of bushing isolator at increase voltage.

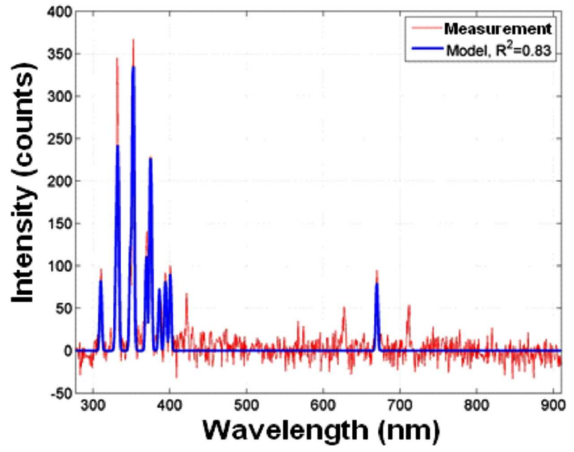

Fig. 7. Spectrum of optical radiation generated by SPDs at increase voltage on the model of bushing isolator, at the voltage $U=0.98 U_{\mathrm{b}}$, obtained from measurements and modeled.

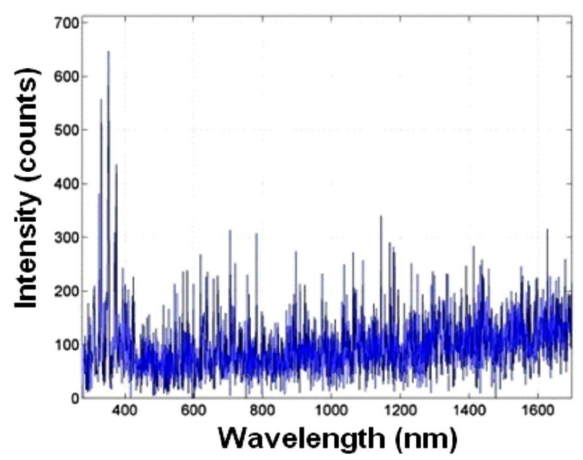

Fig. 8. Spectrum of optical radiation generated by SPDs on the model of bushing isolator at decrease voltage $U=0.98 U_{\mathrm{b}}$.

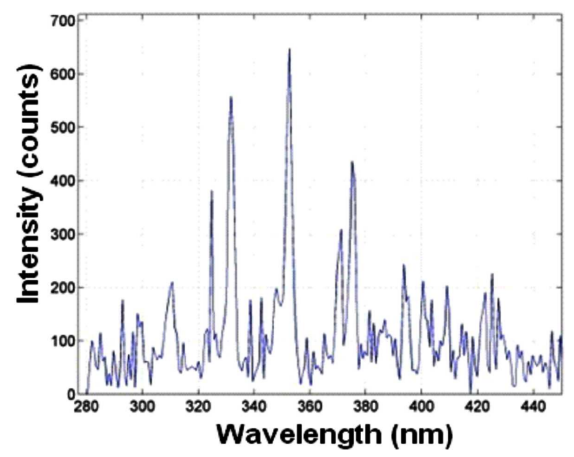

Fig. 9. Spectrum of optical radiation generated by SPDs on the model of bushing isolator at decrease voltage $U=0.98 U_{\mathrm{b}}$.

\section{Spectra of optical radiation emitted by SPDs on a bushing isolator model}

Figure 4 and 5 show the spectra of optical radiation generated by SPDs on the bushing isolator model under test, respectively: for the entire recorded wavelength 


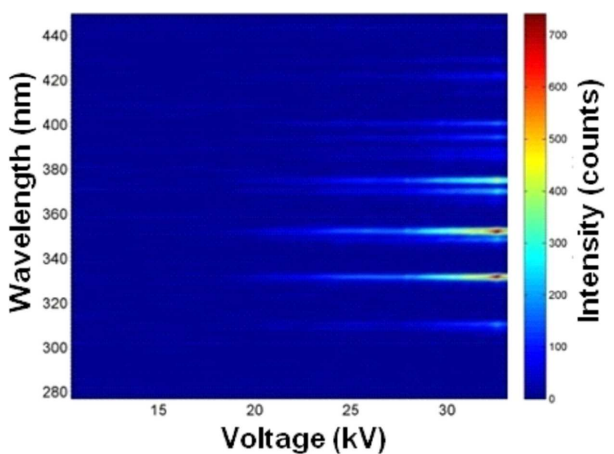

Fig. 10. Changing the intensity of optical radiation generated by the SPDs on the model of bushing isolator at decrease voltage.

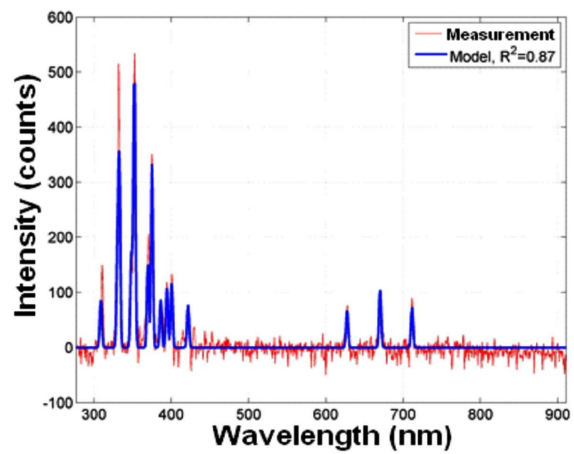

Fig. 11. Spectrum of optical radiation generated by SPDs at decrease voltage on the model of bushing isolator, at the voltage $U=0.98 U_{\mathrm{b}}$, obtained from measurements and modeled.

range $\lambda=270-1700 \mathrm{~nm}$ (Fig. 4) and for the range 270-450 nm including the dominant wavelengths of highest intensity (Fig. 5). The spectra were determined for the optical radiation emitted by increasing the supply voltage, by relative voltage value equal to $0.98 U_{\mathrm{b}}$.

Figure 6 depicts changes in the intensity of radiation registered with the increase in supply voltage from $0.8 U_{\mathrm{b}}$

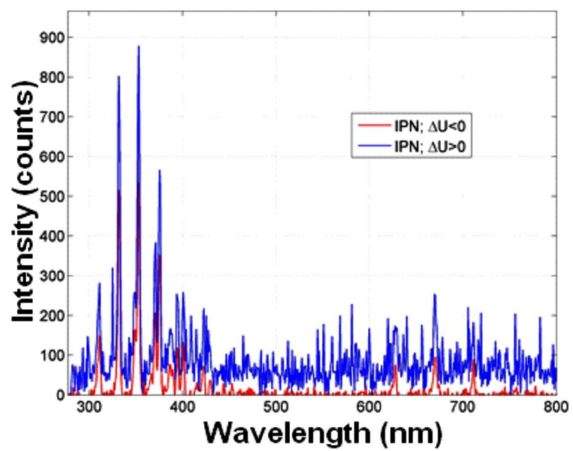

Fig. 12. Comparison of optical radiation spectra generated by SPDs at decrease voltage on the model of bushing isolator, at the voltage $U=0.98 U_{\mathrm{b}}$.

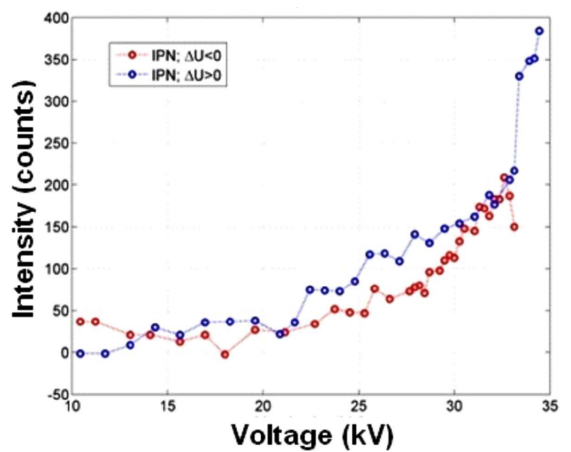

Fig. 13. Changing the intensity of optical radiation generated by the SPDs on the model of bushing isolator for accretion and decrease voltage, for dominant wavelength $\lambda=375 \mathrm{~nm}$.

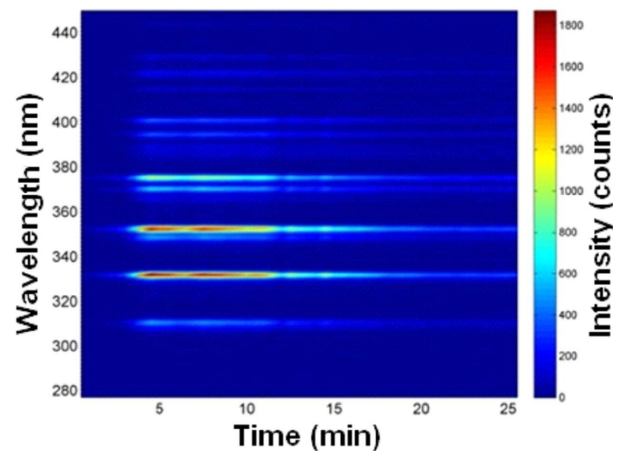

Fig. 14. Changing the intensity of optical radiation generated by the SPDs on a model of bushing isolator as a function of time at the voltage $U=0.98 U_{\mathrm{b}}=$ const.

$(37 \mathrm{kV})$ to $0.98 U_{\mathrm{b}}(45 \mathrm{kV})$. For lower voltage values the registered optical radiation intensity was relatively smaller and negligible, thus it was not taken into account in the characteristics. Figure 7 shows the approximating function estimated for the optical radiation spectrum in the range from 270 to $990 \mathrm{~nm}$, for which the determination coefficient equals $R^{2}=0.9$. In addition, in Fig. 6 numerical values of dominant radiation wavelengths are depicted.

The results obtained by decreasing the supply voltage, are illustrated in analogical manner as with voltage increase, and are shown in Figs. 8-11.

For comparison aims, in Fig. 12 the optical radiation spectrum obtained by voltage decrease and increase, estimated for a relative voltage value equal to $0.98 \mathrm{U}_{\mathrm{b}}$, is presented.

In Fig. 13 changes in the intensity of the optical radiation registered for the dominant wavelength $\lambda=375 \mathrm{~nm}$, for voltage changes in the range from 0.8 to $0.98 U_{\mathrm{b}}$, comparatively, with its lowering and raising, are presented.

Figure 14 shows changes in the intensity of optical radiation generated by SPDs as a function of time $(25 \mathrm{~min})$ at a constant voltage equal to $0.98 U_{\mathrm{b}}$. 


\section{Conclusion}

Based on measurements performed under laboratory conditions, related to registration of optical radiation emitted by SPDs occurring on a bushing insulator, and on analysis of results obtained the following conclusions were formulated:

- Character of optical radiation spectra obtained does not depend on the SPDs voltage generation value. This applies to the voltage range from the discharge ignition (about $0.5 U_{\mathrm{b}}$ ) to a value equal to $0.98 U_{\mathrm{b}}$. For lower voltages (less than $0.8 U_{\mathrm{b}}$ ) the intensity of radiation emitted by SPDs is much smaller, it is characterized by low stability, and the examined phenomenon is strongly stochastic.

- By examination of the impact of lowering and raising the power supply value, no effects on the nature of the obtained spectral dependences was shown. This applies both to the nature of the optical radiation spectra obtained, as well as the wavelength of maximum intensity.

- The spectra determined have two visible extreme values of intensity: at the range $300-430 \mathrm{~nm}$ and 500 $720 \mathrm{~nm}$, respectively, wherein the intensity of radiation in the ultraviolet region (especially UV-A $315-400 \mathrm{~nm}$ ) is significantly higher than in the visible range $380-780 \mathrm{~nm}$.

- In the calculated spectra of the optical radiation, there are three dominant wavelengths: $333 \mathrm{~nm}, 352 \mathrm{~nm}$, and $375 \mathrm{~nm}$, whose intensity is definitely the largest. The intensity of other waves in the two selected bands is more than twice smaller.

- The nature of estimated spectra of the optical radiation does not change over time for a given constant SPDs voltage generation.

- The intensity of optical radiation spectra were modeled using series of Gaussian functions

$$
I(f)=\sum_{i=1}^{N} A_{i} \mathrm{e}^{-\left(\frac{f-f_{i}}{d}\right)^{2}},
$$

where $A_{i}$ is the amplitude of the stria component of length $f_{i} / d, d=1.8$ is an assumed width of a single stria, $f$ is the wavelength.
The average value of each component determines the component length of radiation wave. The applied models are characterized by high degree of compliance of the determination coefficient $R^{2}$, above 0.8 .

\section{References}

[1] P. Bârmann, S. Kröll, A. Sunesson, J. Phys. D, Appl. Phys. 30, 856 (1997).

[2] P. Bârmann, S. Kröll, A. Sunesson, J. Phys. D, Appl. Phys. 29, 1188 (1996).

[3] P. Bârmann, A. Sunesson, in: Conf. Record of the 12th ICDL, Roma (Italy), 1996, p. 197.

[4] A. Beroual, Jpn. J. Appl. Phys. 32, 5615 (1993).

[5] T. Boczar, D. Zmarzły, IEEE Trans. DEIS 13, 632 (2006).

[6] K. Bohnert, P. Gabus, J. Kostovic, H. Brändle, Opt. Lasers Eng. 43, 511 (2005).

[7] S. Conesa, S. Palanco, J.J. Laserna, Spectrochim. Acta Part B 59, 1395 (2004).

[8] J. Deng, H. Xiao, W. Huo, M. Luo, R. May, A. Wang, Y. Liu, Opt. Laser Technol. 33, 305 (2001).

[9] A.S. Farag, M.H. Shewhdi, X. Jin, C. Wang, T.C. Cheng, X. Dong, S. Gao, W. Jing, Z. Wang, Electric Power Systems Res. 50, 47 (1999).

[10] M. MacAlpine, Z. Zhiqiang, M.S. Demokan, Electric Power Systems Res. 63, 27 (2002).

[11] K. Shimizu, T. Oda, Science Technol. Adv. Mater. 2, 577 (2001).

[12] T. Boczar, S. Borucki, A. Cichoń, M. Lorenc, Arch. Acoust. 31, 325 (2006).

[13] F. Witos, A. Olszewska, Acta Phys. Pol. A 118, 1267 (2010).

[14] T. Boczar, P. Frącz, D. Zmarzły, Phys. Chem. Solid State 4, 729 (2003).

[15] P. Frącz, Pomiary Automatyka Robotyka R14, 90 (2010) (in Polish).

[16] P. Fracz, Pomiary Kontrola Automatyka 59, 383 (2011) (in Polish).

[17] S. Sakamato, H. Yamada, S. Sakamoto, H. Yamada, IEEE Trans. DEIS 15, 171 (1980). 